\title{
Correlation of obesity and osteoporosis: Effect of free fatty acids on bone marrow-derived mesenchymal stem cell differentiation
}

\author{
SHAN LV*, LIN WU*, PENG CHENG, JING YU, AISEN ZHANG, JUANMIN ZHA, JUAN LIU, \\ LONG WANG, WENJUAN DI, MIAO HU, HANMEI QI, YUJIE LI and GUOXIAN DING
}

Department of Geratology, The First Affiliated Hospital of Nanjing Medical University, Nanjing 210029, Jiangsu, P.R. China

Received February 10, 2010; Accepted June 3, 2010

DOI: 10.3892/etm_00000095

\begin{abstract}
Studies on the relationship between obesity and bone have recently become widespread. The aim of this study was to investigate the effect of obesity on bone, utilizing a dietinduced obese mouse model, and to explore the role of free fatty acids (FFAs) in the osteogenesis/adipogenesis of mouse bone marrow-derived mesenchymal stem cells (BMSCs). An obese mouse model was established by a high-fat diet (HFD). Proximal femurs were collected at sacrifice, and bone mineral density (BMD) in the proximal femurs was measured by dual-energy X-ray absorptiometry. Bone histomorphometry was performed using undecalcified sections of the proximal femurs. The effect of obesity on the differentiation of mouse BMSCs was assessed by colony formation assays and gene expression analysis. In vitro, various osteogenic and adipogenic genes were determined by real-time quantitative PCR in mouse BMSCs after exposure to conditioned medium (CM) from FFA-treated 3T3-L1 adipocytes. Western blotting was further performed to analyze the representative protein expression of PPAR $\gamma$ and Runx2. BMD and trabecular thickness were significantly greater in the HFD mice than in the control mice. CFU-osteo assay showed significantly increased osteogenesis of BMSCs. The mRNA level of Runx2 was significantly higher, while PPAR $\gamma$ and Pref-1 were significantly lower in BMSCs from the HFD mice compared to the control mice. In mouse BMSCs, the Sox 9 and Runx 2 genes were significantly up-regulated after exposure to $\mathrm{CM}$ from FFA-treated adipocytes, while PPAR $\gamma$ and CEBP- $\alpha$ were significantly down-regulated. Osteogenesis was significantly increased, while adipogenesis was significantly decreased. In conclusion, HFD-induced obesity may play a protective role
\end{abstract}

Correspondence to: Dr Guoxian Ding, Department of Geratology, The First Affiliated Hospital of Nanjing Medical University, 300 Guangzhou Road, Nanjing 210029, Jiangsu, P.R. China

E-mail: dinggx@njmu.edu.cn

${ }^{*}$ Contributed equally

Key words: mesenchymal stem cells, free fatty acids, obesity, osteogenesis, adipogenesis in bone formation by concomitantly promoting osteogenic and suppressing adipogenic differentiation of BMSCs through factors secreted by FFA-treated adipocytes.

\section{Introduction}

Obesity and osteoporosis, two disorders of body composition, are growing in prevalence. Obesity is not only a state of increased adipose tissue, but also a state of chronic low-grade inflammation, characterized by macrophage infiltration (1). Osteoporosis is another highly prevalent disease characterized by accelerated bone resorption and attenuated bone formation, resulting in progressive loss of mineralized bone and a reduction in both bone quantity and quality $(2,3)$. Recently, several studies have provided evidence suggesting their correlation. The traditional view is that obesity is detrimental to bone health, and a negative correlation between body weight or body mass index and bone mass has been reported $(4,5)$. Challenging this widely held view, numerous studies have provided evidence to the contrary, indicating that adipose tissue protects the skeleton (6,7). Recently, the Gilsanz laboratory found that, in young adults, subcutaneous adipose tissue is directly related to bone size and density, whereas visceral adipose tissue is inversely correlated with bone mass (8). Given these discrepancies in results, further investigations aimed at elucidating the relationship between bone and adipose tissue are warranted.

High-fat diet (HFD)-induced obesity in animal models is believed to best mimic the physiological functions of an obese body. Animal experiments supporting the influence of obesity on bone formation in terms of bone marrow mesenchymal stem cell (BMSC) differentiation are limited. It is well-known that the developmental fate of BMSCs is largely determined by the expression of specific transcription factors that act as molecular switches to drive differentiation (9). However, it remains to be determined whether adipose tissue regulates specific transcription factors in BMSC differentiation.

In the present study, a HFD obese mice model was established, and BMSC differentiation between normal control (NC) and HFD mice was compared systematically. The aim was to determine whether adipocytes are an important source of factors that influence bone formation and, most importantly, whether dietary components, particularly free fatty acids, (FFAs) act as circulating regulators of BMSCs. 


\section{Materials and methods}

Reagents. FFAs (palmitic and oleic acid) were purchased from Sigma-Aldrich (St. Louis, MO, USA). FFA-free BSA was purchased from Wako (Japan). 3-Isobutyl-1-methylxanthine, dexamethasone and insulin were also purchased from Sigma. TRIzol was purchased from Invitrogen (San Diego, CA, USA). Moloney murine leukemia virus reverse transcriptase (M-MLV), dNTP, RNase inhibitor and other reverse transcription agents were purchased from Promega Corp. (USA). Anti-murine PPAR $\gamma$ and Runx2 antibodies were purchased from Abcam Corp. (USA).

Animals. C57BL/6 mice (male, 2-week-old) were obtained from Slac Laboratories (Shanghai, China) and randomly divided into normal control (NC) $(n=15)$ and HFD $(n=15)$ groups. Mice in each group were respectively fed normal chow or a HFD for 24 weeks continuously. The mice were maintained under a 12-h light/dark cycle, housed three or four per cage, and had ad libitum access to water. All mice were maintained under humane conditions according to the regulations of the Home Office Animals (Scientific Procedures) Act 1986. The experimental design of this animal study was approved by the Nanjing Medical University Animal Care and Use Committee.

PIXImus measurements. After the femurs were fixed with $4 \%$ paraformaldehyde, bone mineral density (BMD) (total and distal part of femurs) was measured using the PIXImus small animal dual-energy X-ray absorptiometry (DEXA) system (GE Medical System Lunar, Madison, WI, USA).

Histomorphometry. The right proximal femur metaphysis (PFM) was opened to expose the marrow cavity using an isometric low speed saw (Buechler Ltd., USA), and was subsequently fixed in $10 \%$ phosphate-buffered formalin for $24 \mathrm{~h}$. It was then dehydrated in ethanol, defatted in xylene and embedded undecalcified in methylmethacrylate. The frontal sections were cut into 5- $\mu \mathrm{m}$ sections with a microtome (Leica RM2155, Germany), then stained with Goldner's Trichrome staining and Masson-Goldner Trichrome staining for static histomorphometric measurement. A semi-automatic digitizing image analysis system (Osteometrics, Inc., Decatur, GA, USA) was used for quantitative bone histomorphometric measurements. The studied region was cancellous bone between 0.25 and $2 \mathrm{~mm}$ distal to the growth plate-epiphyseal junction. Regions 0.5 and $1 \mathrm{~mm}$ distal to the growth plate of PFM were omitted in order to exclude the primary spongiosa. Static measurements included total tissue area, trabecular area, trabecular perimeter, osteoclast number and osteoclast surface perimeter. These parameters were used to calculate trabecular number, trabecular thickness, trabecular separation, percent osteoclast perimeter and osteoclast number. Histomorphometric parameters were calculated and expressed according to guidelines of the ASBMR Nomenclature Committee (10).

Mouse bone marrow mesenchymal stem cell cultures. BMSCs were obtained from the bone marrow of the mouse femurs. The marrow cavity was flushed with $\alpha$-MEM (Invitrogen), and the marrow was centrifuged at 1,000 rpm for $10 \mathrm{~min}$. The cell pellet was suspended in a basal culture medium containing $10 \%$ fetal bovine serum, $100 \mathrm{U} / \mathrm{ml}$ penicillin and $100 \mathrm{mg} / \mathrm{ml}$ streptomycin, and the cells were then seeded into 6-well plates at a concentration of $1-3 \times 10^{5}$ cells/well. Bone marrow cells were incubated at $37^{\circ} \mathrm{C}$ in a $5 \% \mathrm{CO}_{2}$ humidified atmosphere, and the medium was replaced every 3 days. Non-adherent cells were removed when the medium was replaced. Second passage cells were used for the experiment.

Colony formation assay. For the CFU-osteo assay, BMSCs were grown in basal culture medium for 6 days. The cells were then grown in an osteogenesis-inducing culture medium that comprised basal culture medium supplemented with $10^{-7} \mathrm{M}$ dexamethasone, $0.2 \mathrm{mM}$ ascorbic acid phosphate and $10 \mathrm{mM}$ $\beta$-glycerophosphate (Sigma-Aldrich). After 21 days, the cells were fixed with $10 \%$ formalin and stained with $0.1 \%$ alizarin red, then the number of positive colonies was counted under a light microscope. Only colonies in which the majority $(>50 \%)$ of cells were histologically stained were scored as positive.

For the CFU-adipo assay, BMSCs were grown for 21 days in an adipogenesis-inducing culture medium containing basal culture medium supplemented with $10^{-6} \mathrm{M}$ dexamethasone, $0.5 \mathrm{mM}$ isobutyl-methylxanthine and $3.3 \mu \mathrm{l} / \mathrm{ml}$ insulin. Oil Red O was then added to each well in order to stain the accumulated lipid vacuoles of the cells, and used as a marker of intracellular lipid accumulation in cells. The stained cells were counted under a light microscope.

Preparation of adipocyte-condition medium. Mouse fibroblast 3T3-L1 pre-adipocytes were purchased from the Institute of Biochemistry and Cell Biology (Shanghai, China). For adipogenesis, 3T3-L1 pre-adipocytes were grown to confluence in a $60-\mathrm{mm}$ plate and incubated in an adipogenic cocktail $\left(10^{-6} \mathrm{M}\right.$ dexamethasone, $0.5 \mathrm{mM}$ isobutylmethylxanthine and $3.3 \mu \mathrm{g} / \mathrm{ml}$ insulin) for 2 days. This was followed by incubation in insulinsupplemented medium for an additional 4 days. The cells were transferred to normal medium on Day 7. Palmitic acid and oleic acid were mixed with FFA-free BSA at a weight ratio of 2:1 to produce BSA-bound FFAs. The 3T3-L1 adipocytes were serum-starved overnight in 0.1\% BSA DMEM (Invitrogen) and were then treated with BSA-bound FFAs. FFAs were added to the supernatant from Day 0 to Day 4 of the period of adipogenesis. The mature adipocytes were harvested from the conditioned medium (CM) from Day 8 to Day 10 .

Adipocyte-CM was prepared as follows: confluent cells were washed and maintained in serum-free DMEM medium with $0.2 \%$ BSA without any growth factors for $24 \mathrm{~h}$. Then, the medium was collected and centrifuged $(11,000 \mathrm{rpm}$ for $10 \mathrm{~min}$ at $4^{\circ} \mathrm{C}$ ) to remove cell debris. The FFA-treated CM is referred to as FCM.

RNA preparation and quantitative real-time PCR. Total RNA was extracted from the cultured cells using TRIzol and quantified using real-time PCR. Total RNA $(2 \mu \mathrm{g})$ was reversetranscribed with 200 units M-MLV (Promega, Madison, WI, USA) in the presence of $0.5 \mathrm{mmol} / \mathrm{l}$ deoxynucleotide triphosphate, 25 units RNase inhibitor and $0.5 \mu \mathrm{g}$ N9 random primers, in a total volume of $25 \mu \mathrm{l}$. PCR primers were designed by Primer5 software (Table I). Each quantitative real-time PCR was carried out in triplicate in a $25 \mu \mathrm{l}$ volume of SYBR Green 
Table I. Primer sequences for real-time PCR.

\begin{tabular}{|c|c|c|}
\hline Gene & Forward primer & Reverse primer \\
\hline$\beta$-actin & TAAAGACCTCTATGCCAACACAGT & CACGATGGAGGGGCCGGACTCATC \\
\hline $\operatorname{PPAR} \gamma$ & GACCACTCGCATTCCTTT & CCACAGACTCGGCACTCA \\
\hline $\mathrm{C} / \mathrm{EBP} \alpha$ & CAGTTTGGCAAGAATCAGAGCA & GGGTGAGTTCATGGAGGAATGG \\
\hline Pref-1 & GCTTTTTGTGGTGGAGTTTG & GGGTTCTTAGATAGCGTGGT \\
\hline Runx2 & GGTGAAACTCTTGCCTCGTC & AGTCCCAACTTCCTGTGCT \\
\hline Sox 9 & ACCATTTTGAGGGGAT & GAAGGCTAAGGGACAC \\
\hline Osterix & GCCAGGAGTAAAGAATA & AGAGAGACACCCACAGA \\
\hline Dlx 5 & TGAAAAACGGGGAGATGC & GCTGAGAGAGCGGGATGA \\
\hline
\end{tabular}

Forward (F) and reverse (R) primers are listed for each gene in a 5' to 3' direction, as specified.

Real-time PCR Master Mix (Toyobo, Osaka, Japan). The PCR program was as follows: $10 \mathrm{sec}$ at $95^{\circ} \mathrm{C}$, followed by 40 cycles of $30 \mathrm{sec}$ at $94^{\circ} \mathrm{C}, 30 \mathrm{sec}$ at $60^{\circ} \mathrm{C}, 40 \mathrm{sec}$ at $72^{\circ} \mathrm{C}$ and $5 \mathrm{sec}$ at $80^{\circ} \mathrm{C}$, on a plate reader (Rotor Gene-3000; Corbett Research, Sydney, Australia).

The mean value of triplicates for each sample was calculated and expressed as the cycle threshold $(\mathrm{Ct})$. For each gene studied, the extent of gene expression was calculated as the $\Delta \mathrm{Ct}$ : the difference between the $\mathrm{Ct}$ value of the sample and the $\mathrm{Ct}$ value of $\beta$-actin as the endogenous control. The relative expression level was evaluated by the comparative $\Delta \Delta \mathrm{Ct}$ method, and the software tool REST-384 ${ }^{\circ}$. Expression for each gene was arbitrarily set at 1 to facilitate comparison between several groups.

Western blotting for Runx 2 and PPAR $\gamma$. Primary BMSCs were cultured as previously described, then washed and lysed using RIPA buffer [50 mM Tris- $\mathrm{HCl}$ (pH 7.4), $150 \mathrm{mM} \mathrm{NaCl}, 1 \%$ NP40, 0.5\% sodium deoxycholate, $5 \mathrm{mM}$ EDTA, $5 \mathrm{mM} \mathrm{NaF}, 1$ $\mathrm{mM}$ PMSF, $5 \mu \mathrm{g} / \mathrm{ml}$ leupeptine and $5 \mu \mathrm{g} / \mathrm{ml}$ aprotinin]. Aliquots of cell lysates containing $30 \mu \mathrm{g}$ of total proteins were subjected to SDS-PAGE, transferred to a PVDF membrane and immunoblotted with anti-Runx2, PPAR $\gamma$ and GAPDH antibodies.

Statistical analysis. Values are presented as the mean \pm SD. The difference between groups was tested by the two-tailed t-test or rank-sum test according to the distribution of the data. $\mathrm{P}<0.05$ was regarded as a significant difference.

\section{Results}

In vivo study

Establishment of a high-fat diet-induced obese mice model. The physical and metabolic characteristics of the NC and HFD mice are presented in Fig. 1. As expected, body weight (Fig. 1B) and visceral adipose tissue weight (Fig. 1C) were significantly higher in the HFD mice, which had been maintained with HFD for 20 weeks, compared to the NC mice $(\mathrm{P}<0.01)$. To assess the effect of the HFD on glucose homeostasis in adipose tissues, an oral glucose tolerance test was performed (Fig. 1D). The obese mice exhibited sustained hyperglycemia at fasting blood glucose levels and at 15, 30, 60 and $120 \mathrm{~min}$ after glucose treatment by intragastric administration. In particular, the level of sugar in blood was significantly elevated at $15 \min (\mathrm{P}<0.05)$. These results indicate that the HFD-induced obese mice model was successfully established.

BMD evaluations and static bone histomorphometric analysis. Significant differences in the weight of femurs from the HFD mice compared to the control mice were observed $(\mathrm{P}<0.05)$ (Fig. 2A). The BMD in the proximal femurs was examined by DEXA in the NC and HFD mice (Fig. 2B) and was significantly higher in the HFD mice compared to the NC group $(\mathrm{P}<0.01)$. More extensive histomorphometric studies were performed in both groups. Table II documents the static histomorphometric parameters of the femurs, which were fixed in sections and stained with toluidine blue (Fig. 2C) and Masson-Goldner Trichrome (Fig. 2D), both of which showed more bone trabecula in the HFD than in the NC mice. Trabecular thickness was significantly higher in the HFD mice $(\mathrm{P}=0.05)$. The trabecular number and trabecular surface were higher, while the trabecular separation was lower, in the HFD compared to the NC mice, although this was without significance. In addition, no significant differences were detected in any of the parameters related to the bone absorption rate, such as osteoclast number and osteoclast perimeter.

CFU assay and expression of differentiation genes in BMSCs. To investigate the cause of bone structure changes in the HFD mice, primary osteoblasts and osteoclasts were induced from BMSCs and bone marrow monocytes in both the HFD and NC mice. Tartrate-resistant acid phosphatase (TRAP) staining revealed no significant changes in the number of osteoclasts, and real-time PCR showed no significant changes in the mRNA levels of specific genes, including TRAP, integrin- $\alpha$ and cathepsin K expressed by osteoclasts, between NC and HFD mice (data not shown). These results indicate that obesity may have no impact on osteoclasts.

A BMSC differentiation experiment was performed to determine whether the effect of obesity occurred at the level of stem progenitor cells, which are able to differentiate into osteoblasts and adipocytes (11). As shown in Fig. 3A and B, there was a significantly increased CFU-osteo number (stained by alizarin red) in the HFD mice $(\mathrm{P}<0.01)$. As for $\mathrm{CFU}$-adipo, no statistical difference was found between the HFD and NC groups (data not shown). 
A

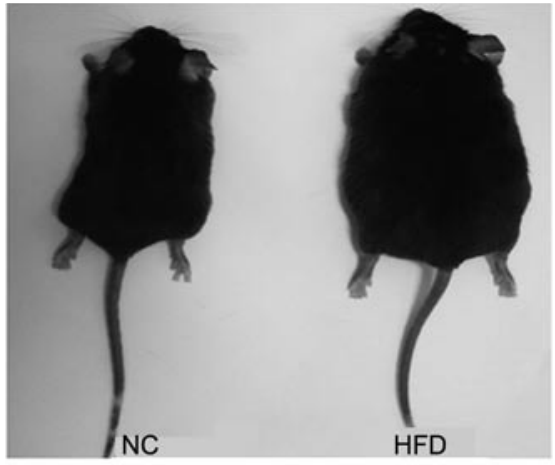

C

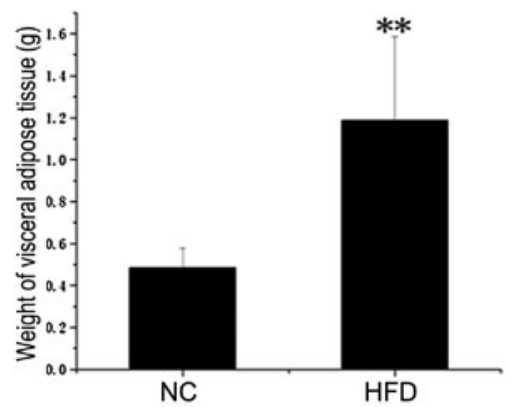

B

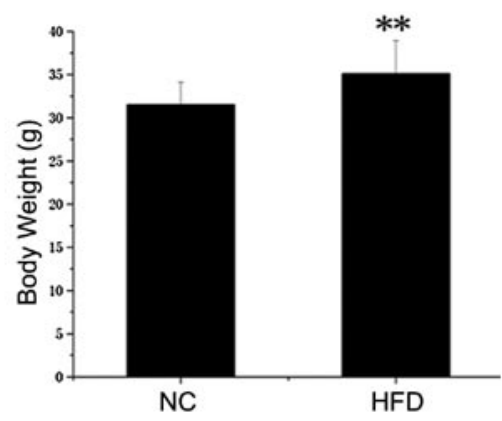

D

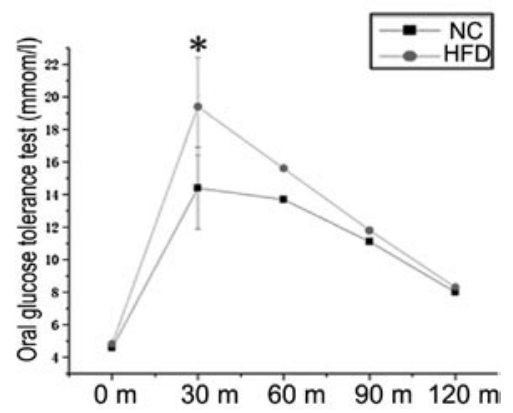

Figure 1. Physiological and metabolic characteristics of the normal control (NC) and high-Fat diet (HFD) mice. (A) Control and HFD mice at 24 weeks of age. The latter had been maintained with a HFD for 20 weeks. Body weight (B) and visceral adipose tissue weight (C) were significantly higher in HFD vs. NC mice $\left(\mathrm{n}=10,{ }^{* *} \mathrm{P}<0.01\right)$. (D) Oral glucose tolerance test. HFD mice showed increased glucose tolerance compared to controls $(\mathrm{n}=6)$ and the blood glucose level reached the maximum at $15 \mathrm{~min}\left({ }^{*} \mathrm{P}<0.05\right)$.

Table II. Static histomorphometry.

\begin{tabular}{lccc}
\hline Bone parameter & Normal control mice & High-fat diet mice & P-value \\
\hline Trabecular surface $(\%)$ & $11.223 \pm 2.658$ & $12.814 \pm 2.313$ & NS \\
Trabecular thick $(\mu \mathrm{m})$ & $43.161 \pm 3.143$ & $48.498 \pm 4.946$ & 0.05 \\
Trabecular no. $($ no. $/ \mathrm{mm})$ & $2.581 \pm 0.479$ & $2.659 \pm 0.522$ & NS \\
Trabecular separation $(\mu \mathrm{m})$ & $356.901 \pm 84.585$ & $339.321 \pm 70.534$ & NS \\
Osteoclast no. $($ no. $/ \mathrm{mm})$ & $0.275 \pm 0.137$ & $0.254 \pm 0.172$ & NS \\
Osteoclast perimeter $(\%)$ & $0.115 \pm 0.049$ & $0.128 \pm 0.089$ & NS
\end{tabular}

Values are presented as the mean $\pm \operatorname{SEM}(n=6)$. NS, not significant.

In order to analyze the effect of obesity on the osteogenic and adipogenic potential of BMSCs at the molecular level, the expression levels of several osteogenic (Sox9, Runx2, Osterix and Dlx5) and adipogenic (PPAR $\gamma$, Pref-1 and $\mathrm{C} / \mathrm{EBP} \alpha)$ genes were determined by real-time PCR. As shown in Fig. 3C and $\mathrm{D}$, the expression level of Runx 2 was significantly higher, while the mRNA transcripts of PPAR $\gamma$ and Pref- 1 were significantly lower, in BMSCs from the HFD group compared to the NC group. As the prime regulator of BMSC differentiation, the protein expression of PPAR $\gamma$ in BMSCs from the $\mathrm{NC}$ mice was higher than that from the HFD mice (Fig. 4E), which coincided with PPAR $\gamma$ gene expression.

In addition, the activity of alkaline phosphatase (ALP) was assayed in isolated murine calvariae osteoblasts. Higher ALP activity in the HFD mice than in the NC mice was noted, although this was not significant (data not shown). The mRNA levels of BMP2 and COLIa1 in osteoblasts analyzed by realtime PCR were also higher in the HFD vs. the NC mice, although this was without significance (data not shown).

\section{In vitro study}

Direct and indirect effect of FFAs on mouse BMSCs. The diet administered to the HFD mice contained mainly FFAs, therefore BMSCs were directly stimulated by FFAs for $48 \mathrm{~h}$ (Fig. 4A and B). Compared to the control, direct treatment of FFAs significantly decreased only the expression level of $\operatorname{PPAR} \gamma(\mathrm{P}<0.05)$.

To obtain further insight into whether adipocytes are an important source of factors acting as circulating regulators of BMSCs, FCM was applied to the BMSCs in vitro for 24 and $48 \mathrm{~h}$. As early as $24 \mathrm{~h}$ after the initiation of treatment, a significant decrease in specific adipogenic genes and an 
A

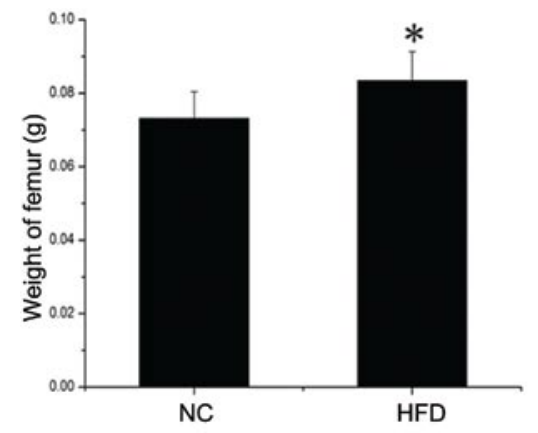

C

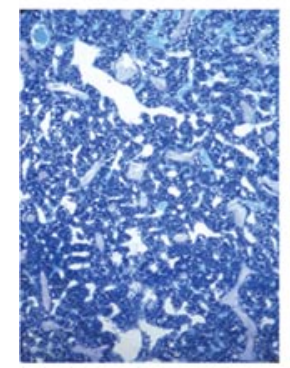

NC

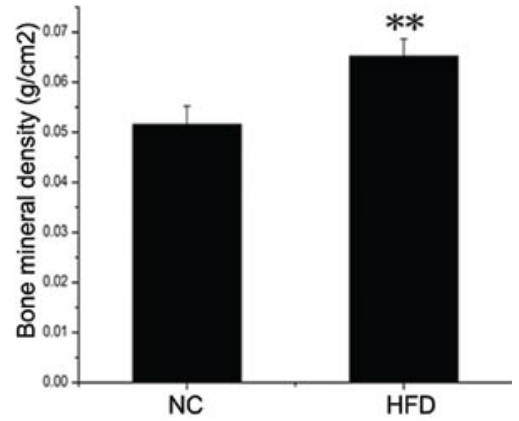

D

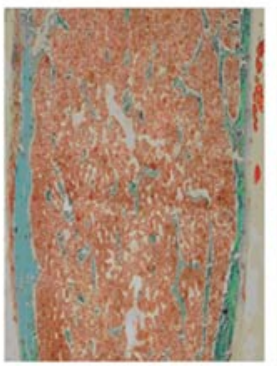

NC

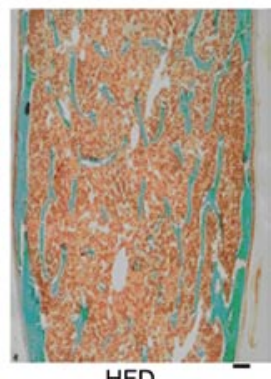

HFD

Figure 2. Evaluation of bone mineral density (BMD) and static bone histomorphometric analysis. Femur weight (A) and BMD (B) measured in proximal femurs by DEXA were significantly higher in HFD vs. NC mice $(n=6),{ }^{*} \mathrm{P}<0.05 ;{ }^{* *} \mathrm{P}<0.01$. Histological sections stained with toluidine blue (C) and Masson-Goldner Trichrome (D), which showed increased bone trabecula in HFD mice. Scale bar, $250 \mu \mathrm{m}$.

A

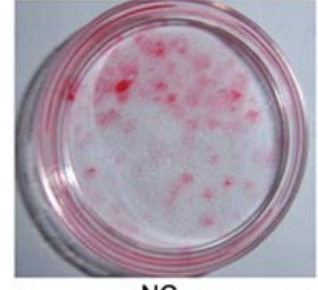

NC

B

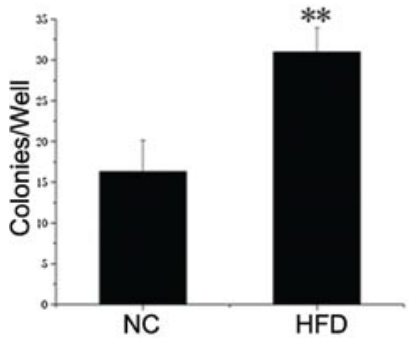

D

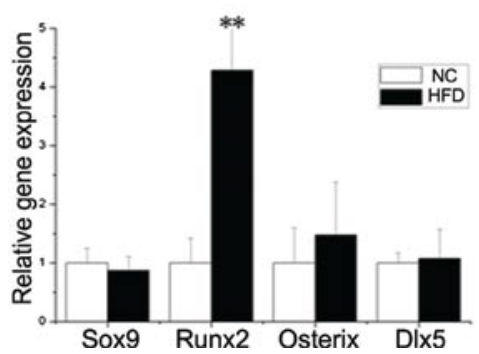

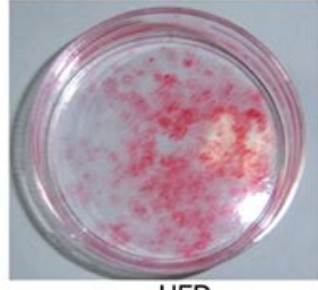

HFD

C

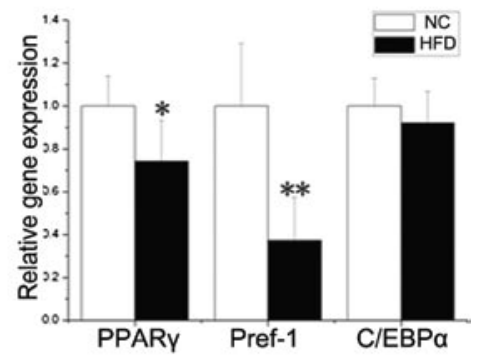

$\mathbf{E}$

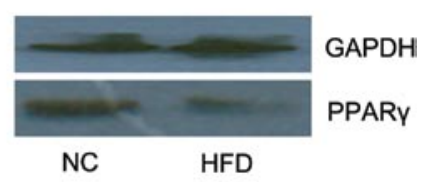

Figure 3. BMSC differentiation assays. BMSCs were obtained from the tibias and femurs of HFD as well as NC mice. (A and B) BMSCs were cultured in basal culture medium, which was then replaced with an osteogenic-inducing culture medium. On Day 21, the cultures were stained with alizarin red (A), and the number of positive colonies (CFU-osteo) was counted (B). The number of CFU-osteo colonies formed from the BMSCs of HFD mice was significantly higher than that formed from the control BMSCs $\left(n=3,{ }^{* *} \mathrm{P}<0.01\right)$. (C and D) mRNA levels of adipogenic and osteogenic genes in BMSCs from NC and HFD mice. BMSCs were harvested after a 8-day culture, then RNA was extracted and gene expression was determined by real-time PCR. mRNA transcripts of PPAR $\gamma$ and Pref-1 (C) were significantly lower in BMSCs from HFD compared to NC mice. The expression level of Runx2 (D) was significantly higher. The relative expression level of each gene was normalized to $\beta$-actin. Data the means of three independent experiments conducted in triplicate $\left({ }^{*} \mathrm{P}<0.05\right.$; ${ }^{* *} \mathrm{P}<0.01$ ). (E) Protein levels of PPAR $\gamma$ in BMSCs from NC and HFD mice. BMSCs were harvested after an 8-day culture. Total proteins were collected and PPAR $\gamma$ was detected by Western blotting. GAPDH was used as a loading control. PPAR $\gamma$ production in BMSCs from NC mice was higher than production in BMSCs from HFD mice. 


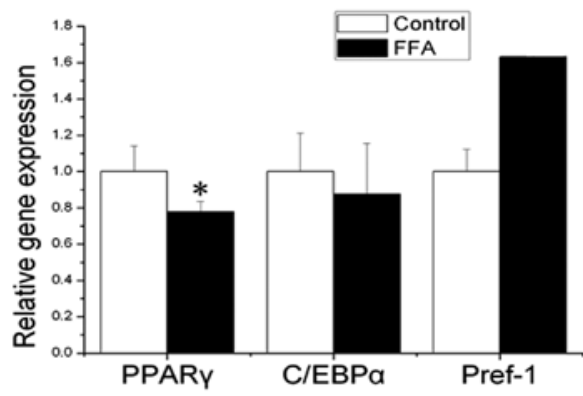

C

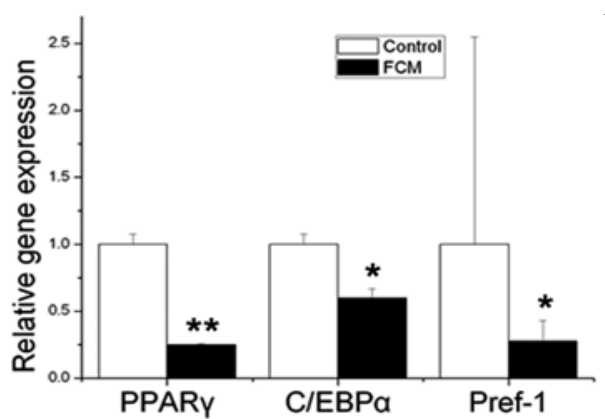

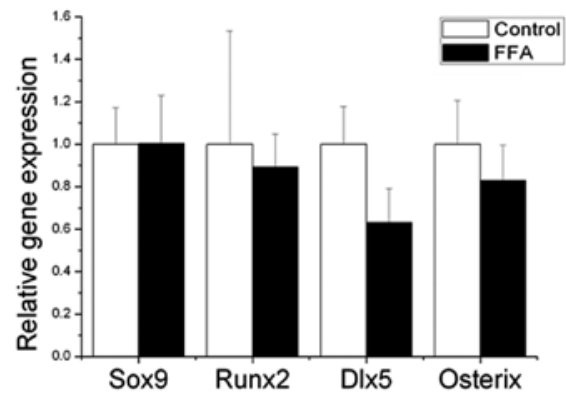

D

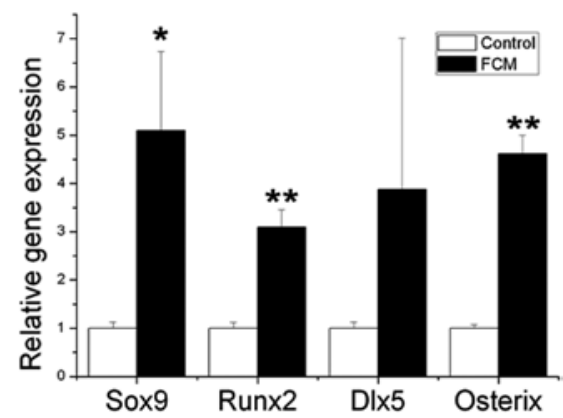

Figure 4. Effect of FFAs and CM from 3T3-L1 adipocytes stimulated by FFAs (FCM) on the gene expression levels of adipogenic and osteogenic genes of mouse BMSCs. BMSCs were obtained from the bone marrow of femurs in 12-week-old C57BL6 mice. After a 48-h treatment with FFAs (A and B) and FCM (C and D), RNA was extracted and adipogenic (A and C) and osteogenic (B and D) genes were determined by real-time PCR. FFA vehicle was used as the control. Compared to the control, direct treatment with FFAs only significantly decreased the expression level of PPAR $\gamma$. However, when BMSCs were exposed to FCM (indirect treatment), the expression levels of PPAR $\gamma, \mathrm{C} / \mathrm{EBP} \alpha$ and Pref-1 were all significantly decreased (C), while the expression levels of Sox9, Runx2 and Osterix were significantly increased (D). The relative expression level of each gene was normalized to $\beta$-actin. Data are the means of three independent experiments conducted in triplicate $\left({ }^{*} \mathrm{P}<0.05 ;{ }^{* *} \mathrm{P}<0.01\right.$ compared to control).

A

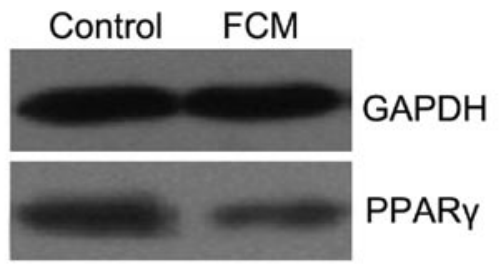

B

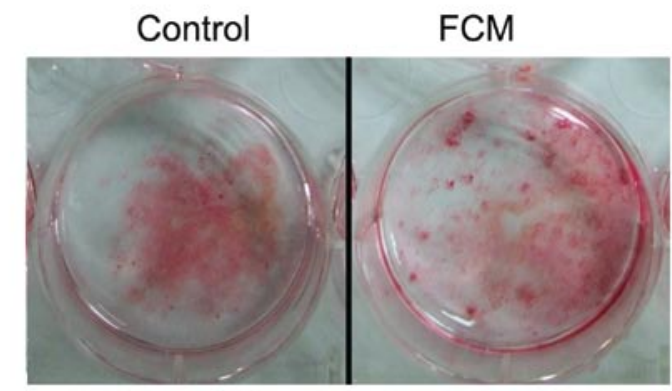

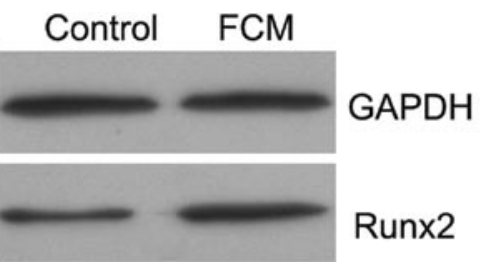

C

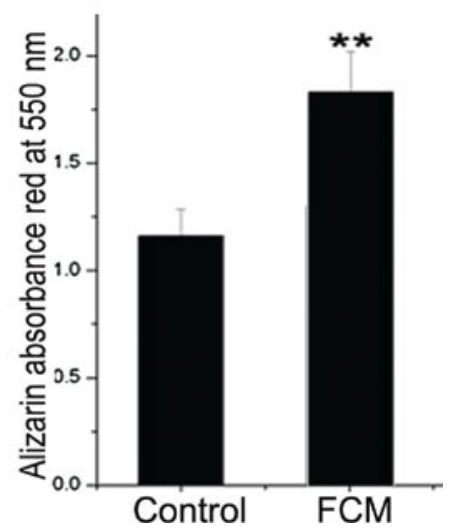

Figure 5. Effect of FCM on the protein expression of PPAR $\gamma$ and Runx2 and the mineralization of mouse BMSCs. BMSCs were obtained from the bone marrow of tibiae and femurs collected from 12-week-old C57BL6 mice. (A) After a 48-h treatment with FCM, total proteins were collected, and PPAR $\gamma$ and Runx2 levels were detected by Western blotting. Normal culture medium was used as the control. GAPDH was used as a loading control. (B) BMSCs were cultured for mineralization, during which FCM was added continuously. After induction for 21 days, the mineral nodus was stained by alizarin red. (C) The red stain was quantified by ethylpyridium chloride extraction, which was measured by the absorbance at $550 \mathrm{~nm}$. The results indicated that FCM promoted BMSC mineralization. ${ }^{* *} \mathrm{P}<0.01$ compared to control.

increase in the early specific osteogenic gene Sox9 was observed (data not shown). After the BMSCs were exposed to
FCM for $48 \mathrm{~h}$, the expression levels of PPAR $\gamma, \mathrm{C} / \mathrm{EBP} \alpha$ and Pref-1 were significantly decreased (Fig. 4C) (PPAR $\gamma, \mathrm{P}<0.01$; 
$\mathrm{C} / \mathrm{EBP} \alpha$ and Pref-1, $\mathrm{P}<0.05)$, while the expression levels of Sox9, Runx2 and Osterix were significantly increased (Fig. 4D) (Sox9, $\mathrm{P}<0.05$; Runx2 and Osterix, $\mathrm{P}<0.01$ ).

Effect of FCM on protein expression and mineralization in mouse BMSCs. As the prime adipogenic and osteogenic genes, the protein expression of PPAR $\gamma$ and Runx 2 was analyzed by Western blotting. PPAR $\gamma$ expression was lower and Runx2 expression was higher in the FCM-treated BMSCs compared to the controls (Fig. 5A). BMSCs were also cultured for mineralization, during which FCM was added continuously. After induction for 21 days, the mineral nodus was stained with alizarin red, which was quantified by ethylpyridium chloride extraction by absorbance at $550 \mathrm{~nm}$ (Fig. 5B and C). As a result, FCM was found to significantly promote BMSC mineralization $(\mathrm{P}<0.01)$.

\section{Discussion}

Increased obesity is a major public health concern and a risk factor for many diseases, but determining whether it is beneficial or detrimental to bone health is difficult.

Our data showed that HFD-induced obesity exerts a protective effect in the development of osteoporosis, consistent with the findings of many clinical research studies (12). $\mathrm{BMD}$, the parameter of femur trabecula and the number of CFU-osteo were increased in obese mice, which may have been regulated through the enhancement of BMSC differentiation towards osteoblasts. However, there was no influence on the number and function of osteoclasts. These results indicate that HFD-induced obesity has an impact on osteoporosis at the level of osteoblasts, but not osteoclasts.

Several potential mechanisms have been proposed to explain the relationship between fat and bone mass. One explanation is that a greater fat mass imposes a greater mechanical stress on bone and, in response, bone mass increases to accommodate the greater load. However, only 27 and $38 \%$ of total body weight in white men and women are attributable to fat mass, respectively (13). Therefore, weight-associated gravitational forces associated with increased fat mass may be insufficient to explain the impact of fat mass on bone.

The dietary components fed to the HFD mice contained mainly FFAs, which serve as a major link between HFD and obesity (14). To date, the study of FFAs has focused largely on their roles in glucose metabolism and insulin resistance $(15,16)$. However, in recent years, a novel viewpoint has emerged asserting that certain dietary components, specifically FFAs, may influence bone metabolism (17-19). In this study, BMSCs were directly stimulated by FFAs, but no significant difference was detected in osteogenic or adipogenic genes, and only PPAR $\gamma$ was decreased. However, after the BMSCs were stimulated by CM from mature adipocytes treated with FFAs, a significantly higher expression level of osteogenic genes, including Runx2, Osterix and Dlx5, and a lower expression level of adipogenic genes, including PPAR $\gamma$, Pref- 1 and $\mathrm{C} / \mathrm{EBP} \alpha$, were noted. These results indicate that FFA plays a role in BMSC differentiation mainly in an indirect manner, first by affecting adipocyte hypertrophy and then by impacting BMSC differentiation through the alteration of factors secreted by adipocytes.
Indeed, adipose tissue is an endocrine organ that secretes multiple hormones, cytokines and inflammatory factors. It is likely that the important relationships between bone and obesity are mediated by adipocyte-derived factors that act on bone. However, different factors have various effects on bone. Inflammatory factors and their detrimental effects on vasculature as well as muscle have long been recognized; not surprisingly, they also inhibit bone formation (20). Several studies have shown that leptin directly promotes the differentiation of osteoblasts $(21,22)$. As for adiponectin, it has a negative effect on bone formation due to an indirect induction of osteoclast formation and inhibition of osteoprotegerin production in osteoblasts $(23,24)$.

It is well-known that active factors secreted by adipocytes can be altered when stimulated by FFAs. For example, acute elevation of plasma FFAs activated the pro-inflammatory nuclear factor NF- $\mathrm{KB}$ pathway, which resulted in increased gene expression of TNF- $\alpha$ and MCP-1 (14). Moreover, FFAs decreased the secretion of adiponectin and leptin into medium $(25,26)$. Collectively, this research and our study indicate that adiponectin or other novel factors secreted by FFA-treated adipocytes may played a key role in BMSC differentiation.

In conclusion, our results provide evidence that HFD-induced obesity may play a protective role in bone formation through factors secreted by FFA-treated adipocytes. This supports the hypothesis that adiponectin or other factors, excepting leptin and inflammatory factors, may play a role in concomitantly promoting the osteogenic and suppressing the adipogenic differentiation of BMSCs. These possible factors may function as a suppressor of osteoporosis in future bio-therapy.

\section{Acknowledgements}

This study was supported by grant no. 30900505 from the China National Natural Scientific Foundation.

\section{References}

1. Wellen KE and Hotamisligil GS: Obesity-induced inflammatory changes in adipose tissue. J Clin Invest 112: 1785-1788, 2003.

2. Raisz LG: Pathogenesis of osteoporosis: concepts, conflict, and prospects. J Clin Invest 115: 3318-3325, 2005.

3. Melton LJ III: Adverse outcomes of osteoporotic fractures in the general population. J Bone Miner Res 18: 1139-1141, 2003.

4. Goulding A, Cannan R, Williams SM, Gold EJ, Taylor RW and Lewis-Barned NJ: Bone mineral density in girls with forearm fractures. J Bone Miner Res 13: 143-148, 1998.

5. Nagasaki K, Kikuchi T, Hiura M and Uchiyama M: Obese Japanese children have low bone mineral density after puberty. J Bone Miner Metab 22: 376-381, 2004.

6. Clark EM, Ness AR and Tobias JH: Adipose tissue stimulates bone growth in prepubertal children. J Clin Endocrinol Metab 91: 2534-2541, 2006.

7. Reid IR: Relationships among body mass, its components, and bone. Bone 31: 547-555, 2002.

8. Gilsanz V, Chalfant J, Mo AO, Lee DC, Dorey FJ and Mittelman SD: Reciprocal relations of subcutaneous and visceral fat to bone structure and strength. J Clin Endocrinol Metab 94: 3387-3393, 2009.

9. Beresford JN, Bennett JH, Devlin C, Leboy PS and Owen ME: Evidence for an inverse relationship between the differentiation of adipocytic and osteogenic cells in rat marrow stromal cell cultures. J Cell Sci 102: 341-351, 1992.

10. Parfitt AM, Drezner MK, Glorieux FH, Kanis JA, Malluche H and Meunier PJ: Bone histomorphometry: standardization of nomenclature, symbols, and units. Report of the ASBMR Histomorphometry Nomenclature Committee. J Bone Miner Res 2: 595-610, 1987. 
11. Pittenger MF, Mackay AM, Beck SC, Jaiswal RK, Douglas R, Mosca JD, Moorman MA, Simonetti DW, Craig S and Marshak DR: Multilineage potential of adult human mesenchymal stem cells. Science 284: 143-147, 1999.

12. Reid IR: Relationships between fat and bone. Osteoporos Int 19 : 595-606, 2008.

13. Zhao LJ, Jiang H, Papasian CJ, Maulik D, Drees B, Hamilton J, Deng HW and Zhao LJ: Correlation of obesity and osteoporosis: effect of fat mass on the determination of osteoporosis. J Bone Miner Res 23: 17-29, 2008.

14. Boden G, She P, Mozzoli M, Cheung P, Gumireddy K, Reddy P, Xiang $\mathrm{X}$, Luo $\mathrm{Z}$ and Ruderman $\mathrm{N}$ : Free fatty acids produce insulin resistance and activate the proinflammatory nuclear factor- $\kappa$ B pathway in rat liver. Diabetes 54: 3458-3465, 2005.

15. Soga T, Ohishi T, Matsui T, Saito T, Matsumoto M, Takasaki J, Matsumoto S, Kamohara M, Hiyama H, Yoshida S, Momose K, Ueda Y, Matsushime H, Kobori M and Furuichi K Lysophosphatidylcholine enhances glucose-dependent insulin secretion via an orphan $\mathrm{G}$ protein-coupled receptor. Biochem Biophys Res Commun 326: 744-751, 2005.

16. Yamada C, Yamada Y, Tsukiyama K, Yamada K, Udagawa N, Takahashi N, Tanaka K, Drucker DJ, Seino Y and Inagaki N: The murine glucagon-like peptide-1 receptor is essential for control of bone resorption. Endocrinology 149: 574-579, 2008

17. Grey A: Fatty acids and bone. Clinic Rev Bone Miner Metab 7: 210-215, 2009.

18. Cornish J, MacGibbon A, Lin JM, Watson M, Callon KE, Tong PC, Dunford JE, van der Does Y, Williams GA, Grey AB, Naot D and Reid IR: Modulation of osteoclastogenesis by fatty acids. Endocrinology 149: 5688-5695, 2008.
19. Watkins BA, Li Y, Allen KG, Hoffmann WE and Seifert MF: Dietary ratio of $(n-6) /(n-3)$ polyunsaturated fatty acids alters the fatty acid composition of bone compartments and biomarkers of bone formation in rats. J Nutr 130: 2274-2284, 2000.

20. Morley JE and Baumgartner RN: Cytokine-related aging process. J Gerontol A Biol Sci Med Sci 59: M924-M929, 2004.

21. Steppan CM, Crawford DT, Chidsey-Frink KL, Ke H and Swick AG: Leptin is a potent stimulator of bone growth in ob/ob mice. Regul Pept 92: 73-78, 2000.

22. Frühbeck G: Intracellular signalling pathways activated by leptin. Biochem J 393: 7-20, 2006.

23. Luo XH, Guo LJ, Xie H, Yuan LQ, Wu XP, Zhou HD and Liao EY: Adiponectin stimulates RANKL and inhibits OPG expression in human osteoblasts through the MAPK signaling pathway. J Bone Miner Res 21: 1648-1656, 2006.

24. Oshima K, Nampei A, Matsuda M, Iwaki M, Fukuhara A, Hashimoto J, Yoshikawa $\mathrm{H}$ and Shimomura I: Adiponectin increases bone mass by suppressing osteoclast and activating osteoblast. Biochem Biophys Res Commun 331: 520-526, 2005.

25. Berger J, Tanen M, Elbrecht A, Hermanowski-Vosatka A, Moller DE, Wright SD and Thieringer R: Peroxisome proliferatoractivated receptor- $\gamma$ ligands inhibit adipocyte $11 \beta$-hydroxysteroid dehydrogenase type 1 expression and activity. J Biol Chem 276: 12629-12635, 2001.

26. Shintani M, Nishimura H, Yonemitsu S, Masuzaki H, Ogawa Y, Hosoda K, Inoue G, Yoshimasa Y and Nakao K: Downregulation of leptin by free fatty acids in rat adipocytes: effects of triacsin C, palmitate, and 2-bromopalmitate. Metabolism 49: 326-330, 2000. 